Methods Data on the daily incidence of general illness, tiredness, headaches, fever, muscle aches, runny nose, sore throat, coughing, nausea and eye irritation (symptom complexes) and sheep treatment practices during the week following sheep treatment was obtained by using a self-reported diary. Associations between work practices and the incidence of symptom complexes were investigated by distributed lag models.

Results 352 UK sheep farmers completed the diary. The incidence of all symptom complexes was significantly higher on days 1 and 2 than on day 7 but there was little evidence of any differences in symptom reporting between OP and non-OP users. The number of sheep treated was not linked to the incidence of acute ill-health (whatever lag model was used) except that the number of sheep treated on day 4 was associated with an increased risk of reporting $\geq 2$ symptom complexes in $\mathrm{OP}$ users only.

Conclusions The increase in symptom reporting after the use of both non-OPs and OPs may reflect a common response to pesticides of different modes of action. However, the lack of any association with biologically important exposure lags suggests other treatment related factors may well be important.

\title{
Pesticides
}

43

\section{SYMPTOM REPORTING AMONGST FARMERS USING PESTICIDES TO TREAT SHEEP}

Hanaa Bayomy, ${ }^{1}$ Frank de Vocht, ${ }^{1}$ Huw Rees, ${ }^{2}$ John Thompson, ${ }^{2}$ Lakshman Karalliedde, ${ }^{3}$ Andrew Povey ${ }^{1}$ University of Manchester, Manchester, UK; ${ }^{2}$ Cardiff University, Cardiff, UK; ${ }^{3}$ Guy's \& St Thomas' NHS Foundation Trust, London, UK

10.1136/oemed-2011-100382.43

Objectives Farmers who treat their sheep for ectoparasites with organophosphate $(\mathrm{OP})$ dips report a range of flu-like symptoms known as 'dipper's flu'. The nature and causes of 'dipper's flu' are poorly understood. The aim of this study was to investigate whether $\mathrm{OP}$ exposure was associated with acute ill health. 